\title{
Multilinguales
}

$14 \mid 2020$

Enseignement universitaire et professionnalisation : enjeux, questionnements et défis socio-économiques

\section{Sémiotisation et littérarisation de la focalisation dans le roman Bleu-Blanc-Rouge d'Alain Mabanckou} Semiotization and literarization of focalization in Alain Mabanckou's novel Bleu-Blanc-Rouge

\section{Aboubakar Gounougo}

\section{OpenEdition}

Journals

Édition électronique

URL : https://journals.openedition.org/multilinguales/5798

DOI : $10.4000 /$ multilinguales. 5798

ISSN : 2335-1853

Éditeur

Université Abderrahmane Mira - Bejaia

Référence électronique

Aboubakar Gounougo, «Sémiotisation et littérarisation de la focalisation dans le roman Bleu-BlancRouge d'Alain Mabanckou », Multilinguales [En ligne], 14 | 2020, mis en ligne le 10 décembre 2020, consulté le 02 juillet 2021. URL : http://journals.openedition.org/multilinguales/5798 ; DOI : https:// doi.org/10.4000/multilinguales.5798

Ce document a été généré automatiquement le 2 juillet 2021.

\section{cc)}

Multilinguales est mise à disposition selon les termes de la Licence Creative Commons Attribution Pas d'Utilisation Commerciale - Pas de Modification 4.0 International 


\title{
Sémiotisation et littérarisation de la focalisation dans le roman Bleu- Blanc-Rouge d'Alain Mabanckou
}

\author{
Semiotization and literarization of focalization in Alain Mabanckou's \\ novel Bleu-Blanc-Rouge
}

Aboubakar Gounougo

Le roman au titre évocateur Bleu-Blanc-Rouge d'Alain Mabanckou est le fruit d'une écriture volontairement accessible construite autour des déictiques anaphoriques « je », indice de la première personne verbale et le « il » de la troisième personne dont les effets littéraires de leur enchâssement sont irréductibles dans le discours. Le règne sans partage du pronom de la première personne, sujet de la narration, est très marqué en tant que ce déictique est celui qui génère les diverses occurrences de l'autre pronom anaphorique. Les effets palpables d'une telle organisation scripturaire menée par la première personne narrative se traduisent manifestement par la présence de grands monologues introspectifs, semblables à ceux que l'on rencontre dans l'écriture dramatique et qui structurent le récit de bout en bout.

2 Le récit Bleu-Blanc-Rouge n'est pas une histoire linéaire racontée à la troisième personne par un narrateur à la focalisation tantôt surplombante tantôt externe qu'on chercherait à localiser par rapport à son énoncé, mais plutôt, un récit monologué au cours sinueux, l'œuvre d'un sujet-narrateur en proie constamment à l'introspection. D'ailleurs, le roman de l'écrivain congolais débute par un grand monologue servant d' "ouverture " de type méditatif et s'achève par une "fermeture " du même type discursif. Entre ces deux étapes narratives, se trouve bien évidemment le récit à proprement dit qui, lui-même, est à rayures, parce que traversé constamment par des monologues qui ne font pas que signaler la présence d'un locuteur assumant son discours. Il s'agit d'une véritable technique d'écriture qui privilégie le regard d'une conscience sur le monde extérieur à partir de sa propre vision, avec pour point focal le sujet de la vision interne. Le style d'énonciation qui autorise la focalisation dynamique dans ce récit est redevable de l'usage subtil par le romancier de la deixis anaphorique 
comportant les individus linguistiques « je », subjectif et le « il », objectif. La présente contribution se donne pour point de départ, ce niveau énonciatif de la focalisation, qui dans le fond, est à la base de certaines configurations tant formelles que sémantiques animant le récit. Le jeu de leur alternance est productif de littérarité et nous le prendrons pour prétexte afin de comprendre le travail de l'écrivain qui est parvenu à faire cohabiter par l'écriture, deux mondes métonymiques, celui qui est extérieur au sujet-narrateur, qui s'impose à sa vision, et qu'il tente par ailleurs d'appréhender à partir de son monde intérieur, c'est-à-dire celui où s'exprime son « moi » profond.

3 Il est donc clair ici que les enjeux de cette réflexion seront à la fois d'ordre poétique que stylistique. Et pour que ces enjeux apparaissent clairement au lecteur, nous nous attellerons, d'une part, à proposer une analyse phénoménologique de la sémiotique du point de vue narratif dans le roman d'Alain Mabanckou pour en saisir les traits essentiels et, de l'autre, les implications littéraires afférentes à la vision dynamique du sujet-narrateur dans son acte d'énonciation.

\section{Phénoménologie sémiotique de la vision narrative dans Bleu-Blanc-Rouge}

4 Au premier abord du roman d'Alain Mabanckou, il saute aux yeux du lecteur qu'il s'agit bien d'un récit à la première personne verbale «je» qui autorise l'occurrence du déictique anaphorique «il», l'une des marques de l'objectivation de la narration. La question qui se pose immédiatement après la lecture du roman, est celle, sans doute datée, de savoir s'il est question d'un discours ou d'un récit? Mais, à bien observer les faits relatifs à l'énonciation, cette question qui a perdu quasiment de toute crédibilité du fait des révolutions génériques successives de l'écriture, mérite ici d'être posée, malgré tout, pour faire la lumière sur cet aspect de la vision narrative. Ce récit n'a pas de frontière avec le discours puisque ce dernier type générique est celui dans lequel le premier trouve sa raison d'être sous forme de relation objective des péripéties ou des évènements qui sont, eux, en retour, le catalyseur de ce discours à valeur introspective. Ce mixte énonciatif fait comprendre cette position de Molinié qui s'est toujours gardé de la distinction radicale du récit et du discours : «l'on doit considérer, à un niveau de base que, tout texte comme discours, en tant que production d'un émetteur premier, l'instance énonciative responsable de tout le montage et de toute la succession verbale qui se manifestent dans l'objet-texte » (Molinié, 1993 :23).

5 Il est donc intéressant qu'en tout début d'analyse, l'on décrive les faits d'énonciation qui produisent la vision subjective du sujet-narrateur dont les méditations personnelles lui permettent de révéler des faits et évènements en toute objectivité.

\subsection{Sémiotique de la deixis anaphorique du « je » et du « il »}

6 Dans Bleu-Blanc-Rouge d'Alain Mabanckou, l'énonciation dans son fonctionnement pose nécessairement la question de la vision du sujet-narrateur. Celui-ci se situe bien dans un univers dont il tente de dévoiler au lecteur, tous les contours et paramètres qui se déclinent en une condition d'existence déshumanisante. Le règne du sujet verbal « je » qui médite sur un objet extérieur dont le marqueur grammatical est le pronom «il », est sans appel. Dans le roman d'Alain Mabanckou, il est question d'un personnage qui raconte des faits et évènements dont il est témoin et qu'il tente de rapporter avec un 
minimum d'objectivité ou d'exactitude. Ce personnage est, selon la terminologie de Genette $^{1}$, un narrateur à la fois extradiégétique et intradiégétique parce qu'il raconte extérieurement une histoire et dans celle-ci il joue souvent lui-même le rôle d'un narrateur qui raconte une autre histoire. En plus de ce double rôle de narrateur,

ce personnage comme tous les autres, peut être considéré abstraitement dans son rôle actantiel, ou envisagé comme "acteur", habillé de rôles thématiques et de qualifications qui le mettent en relation avec d'autres personnages de l'histoire : il se situe au niveau « diégétique " à l'intérieur de l'univers fictif produit par le récit et ses effets (Borgomano, $1985: 22)$

7 C'est ce qui explique que de l' « Ouverture » de son récit à sa « Fermeture » en passant par le développement à proprement dit des péripéties, la vision narrative du sujet soit très mobile ou super dynamique. Ainsi, de nombreuses tranches de ce récit finissent par revêtir les marques du discours personnel, le sujet de l'énonciation « je », prenant à chaque instant le dessus sur l'objet de l'énonciation signalé par le pronom «il». Plus intéressant, de nombreux discours virent, sans autre forme de procès, à des monologues introspectifs dans lesquels le sujet-narrateur Massala-Massala alias Marcel Bonaventure alias Éric Jocelyn-George laisse échapper son «moi » profond dans son rapport à un univers impitoyable des hommes, depuis «Le pays » (29) jusqu'à « Paris » (119).

8 La présence incompressible du pronom de la première personne «je ", à la fois personnage principal et sujet-narrateur désignant Massala-Massala, traduit clairement le règne sans partage d'une seule voix narrative. C'est cette dernière qui donne la parole, quand et où elle le veut, aux êtres qui défilent dans son récit et avec lesquels elle partage les mêmes espaces sociaux et les mêmes histoires voire les mêmes vies. Face au pouvoir du déictique «je » qui se comprend aisément du fait de ce récit dont l'étape initiale est peu mouvementée, les péripéties très dramatiques et leur chute pathétique, se trouve le pronom anaphorique « il ». Ses occurrences dans le discours du «je-sujet-narrateur» impliquent l'écriture d'une introspection motivée, parce que provoquée par des faits et évènements qui, comme une fatalité, imposent au sujet de s'interroger de l'intérieur. Le pronom « il » renvoie, en clair, à l'extérieur, cet extérieur construit autour de faits et évènements à mettre à l'actif de personnages types comme ceux de Moki et de Préfet parmi tous ces êtres déshumanisés par les espaces «Le pays ", dans une moindre mesure et "Paris », la capitale française tant convoitée et dans laquelle ils tentent de survivre par tous les moyens, même les plus sordides et donc les plus répréhensibles. Et le mal-être des personnages, le sujet-narrateur en a fait personnellement l'amère expérience. Du point de vue donc de l'énonciation qui soustend la focalisation interne enchâssant à sa structure les visions zéro et externe, l'on peut tirer profit de cette affirmation d'autorité d'Oswald Ducrot et Tzvetan Todorov à propos de la science du narrateur :

le narrateur décrit l'univers mental du personnage de l'intérieur ou de l'extérieur. Dans le cas où il s'introduit dans l'esprit des personnages, ce procédé peut s'appliquer à un seul héros ou à plusieurs; et dans ce dernier cas, le passage d'une conscience à l'autre peut suivre ou non un dessin rigoureux. C'est lorsque le souci de justifier les connaissances du narrateur est réduit au minimum, qu'on parle d'un auteur (ou narrateur) omniscient [...]. On peut distinguer aussi des degrés de «profondeur ", l'inégale pénétration du narrateur (ou encore son angle de vision): il ne décrit que des comportements et se contente d'observer; ou rapporte les pensées du personnage (que celui-ci est supposé seul connaître); ou nous donne connaissance de processus dont le personnage lui-même ignore tout (ambition qui 
se fonde souvent, au $\mathrm{XX}^{\mathrm{e}}$ siècle, sur le fonctionnement de l'inconscient). On peut distinguer également entre les types de connaissances impliqués : psychologiques, événementielles, etc. (Todorov et Ducrot, 1972 :415)

Ce que disent les deux théoriciens est approprié pour comprendre le système énonciatif bâti autour de la présence absolue du pronom de la première personne «je » qui implante face à lui le déictique d'objectivation « il ». Ce deuxième indicateur est utilisé dans le récit pour traduire la présence du monde, celui d'un espace dans lequel d'autres personnages provoquent des faits et évènements qui, tout en étant extérieurs au personnage-narrateur, l'embarquent dramatiquement tout autant qu'il est acteur dans ce monde des autres qui est aussi le sien. Il y a donc dans le roman d'Alain Mabanckou une intimité irrévocable entre les deux indicateurs énonciatifs «je » et «il » qui est, elle-même (cette intimité), l'expression linguistique du rapport naturel de la conscience individuelle à la société qui la légitime. Ces deux marqueurs de la vision narrative ne sont pas isolés dans le récit de l'écrivain congolais. Ils sont nécessairement sujets de prédicats dans les syntagmes dont la concaténation est au fondement du récit dans lequel d'autres indices grammaticaux leur sont tributaires. Ce sont, entre autres, les modalisateurs, les déictiques spatio-temporels, le lexique affectif et les évaluatifs, les compléments d'objets ou de circonstances, la ponctuation, les figures de rhétorique, les verbes avec leurs temps et leurs modalités d'emploi, le rythme, etc... Ces marques scripturaires, parmi tant d'autres, qui contribuent à l'expression tant de la subjectivité que de l'objectivité de l'acte narratif, selon leur usage en rapport avec la deixis individuelle dont il est question ici, relèvent bien de l'énonciation qui, de fait, offre matière à une description caractéristique du point de vue narratif du sujet face à l'objet de la narration. Et ce point de vue du sujet-narrateur n'est pas figé mais plutôt dynamique, la vision interne s'assujettissant les visions zéro et externe et le tout, complexe, a nécessairement une fonction structurante du récit.

\subsection{Fonction structurante de la vision du sujet-narrateur}

10 En suivant le fonctionnement complexe des déictiques anaphoriques, le «je » en tant que sujet-narrateur et le « il » qui désigne l'objet extérieur à ce sujet-narrateur qui le relate de l'intérieur, il ressort que la vision narrative qui découle de ce fonctionnement énonciatif subtil est structurante. Dans le récit, en effet, le sujet-narrateur qui est aussi témoin et partie prenante des évènements et faits qu'il relate, perçoit de son intérieur propre le monde. En revanche, de par la vision zéro qui atteint «au récit nonfocalisé $»^{2}$, et qu'il utilise en des circonstances bien opportunes de son énonciation, le personnage-narrateur tente toujours de dévoiler les moindres détails en les détachant apparemment de son «moi » personnel. Son souci premier est d'atteindre à la perception de l'univers qu'il décrit comme s'il en était le créateur. Quand il décide de s'effacer de la scène, il s'agit toujours de la continuité de la stratégie adoptée afin de relater les faits et évènements avec beaucoup plus de réalisme et donc d'objectivité. Ainsi, dans le récit, de part en part, trois visions intriquées débouchent sur trois types d'énoncés aussi enchevêtrés que les marques de leur énonciation et, comme par hasard, le découpage du roman d'Alain Mabanckou est lui aussi ternaire : une « ouverture », un récit fait des péripéties se déroulant dans les espaces «Le pays » et «Paris » et pour finir, une « fermeture ».

11 Cela dit, quand le sujet-narrateur est absolument présent dans son propre récit qui, par ailleurs, le vise aussi comme l'un de ses objets, il arrive que celui-ci vire du simple 
récit subjectif à un autre type de discours, le monologue qui, bien incorporé, exempte le lecteur de la question de la transgénéricité qui pouvait naître de cette intrication discursive. L'extrait du roman qui ouvre l' «Ouverture » elle-même est représentative de toutes ces tranches de récit ou le fonctionnement énonciatif de l'embrayeur «je » est absolu :

J'arriverai à m'en sortir.

Je ne sais plus de quel côté se lève ou se couche le soleil. Qui entendrait mes plaintes? Je n'ai plus aucun repère ici. Mon univers se limite à ce cloisonnement auquel je me suis accoutumé. Pouvais-je me comporter autrement? J'ai fini par ériger dans mon for intérieur l'espace qui me fait défaut. J'emprunte des voies désertées. Je traverse des villages fantômes. J'entends mes pas sur les feuilles mortes. Je surprends des oiseaux de nuit somnolant sur une patte. Je m'arrête. Je reprends la route jusqu'aux premières lueurs de l'aube... (11)

Nous passons sous silence, pour le moment, les escomptes poétiques et stylistiques internes à chacun des récits d'illustration pour n'y interroger que le caractère structurant de la focalisation. Ainsi, ce récit est un monologue du même type que ceux l'on rencontre dans les textes théâtraux, précisément dramatiques ou tragiques. Il est articulé autour de l'emploi absolu du déictique anaphorique « je » qui désigne le sujetnarrateur en la personne de Massala-Massala alias Marcel Bonaventure. Dans le roman d'Alain Mabanckou, ce type de monologues abonde et commande à tout le récit assumé par le narrateur dont la conscience est éprouvée par les évènements extérieurs, la plupart du temps malheureux et qui s'imposent à lui comme une fatalité. Le souci de rendre avec exactitude ses frasques dont certains des personnages types du roman sont les véritables responsables, pousse le sujet-narrateur à adopter les autres points de vue narratifs où la première personne verbale, tantôt s'efface totalement derrière les faits et les personnages qui les provoquent, tantôt les supplante par son omniscience et son omniprésence camouflée. Il s'agit bien d'une stratégie de relation des faits et évènements, privilégiée par le personnage-narrateur, qui se veut le plus objectif possible pour tenter de convaincre le lecteur de son innocence dans cette condition de médiocrité existentielle ou de déshumanisation révélée par son propre récit. Ainsi, à titre d'illustration, ce deuxième extrait dont la vision narrative est différente de celle du passage précédent :

Boulou était le spécialiste de l'immobilier. On l'appelait à juste titre «l'Agent immobilier ». Comme son sobriquet l'indiquait, il opérait dans le domaine de l'immobilier. Il travaillait avec des compatriotes affublés du surnom de Bulldozers. Ceux-ci avaient pour mission de ratisser les immeubles d'un arrondissement en vue d'y détecter des appartements inoccupés et de les proposer, moyennant une contrepartie financière, aux squatters. Cette rémunération était proportionnelle à la surface du logement à squatter. Les bulldozers agissaient sous le contrôle méticuleux de Boulou, et il préservait jalousement son exclusivité sur le fief. (148)

Le même et unique sujet-narrateur tient ici aussi la parole. Seulement, celle-ci se distingue structurellement de l'énoncé d'illustration précédent du point de vue de la vision narrative qui passe de la sphère du dedans à celle du dehors. Ici donc, nulle trace grammaticale de celui qui dresse le portrait de Boulou, grammaticalement représenté par l'anaphorique «il». Le sujet-narrateur dont on peut toutefois percevoir la silhouette déictique camouflée à travers le pronom « on » objectivement indéterminé, s'est tout simplement effacé ou se tient momentanément à distance de son énoncé. Nous sommes dans un cas typique de focalisation externe qui permet au narrateur d'atteindre à une certaine objectivité par la mise en veilleuse de son regard introspectif à travers la suppression matérielle du déictique anaphorique «je» de la subjectivité. 
Cette deuxième forme de focalisation qui sous-tend certains paliers narratifs inclus dans la vision interne qui domine de bout en bout le roman d'Alain Mabanckou est très voisine $\mathrm{du}$ " récit non-focalisé " $^{3}$ comme dans l'extrait suivant :

\begin{abstract}
Soté le Piocheur était un personnage dont la fourberie se ressentait au premier contact. Il s'accommodait de cette image d'homme méprisable et l'entretenait jusqu'à la caricature. Peut-être ne voulait-il côtoyer que ceux qu'il choisissait, et il se méfait de tout visage étranger.

C'était pour moi le plus antipathique de tous. Aucune humanité. Aucun cœur. Le profit dilatait ses pupilles mauves. Aucun mot ne venait de lui au sujet de ses activités. Grand de taille, avec des sourcils broussailleux en accent circonflexe, le visage ravagé par des boutons purulents qu'il crevait devant ses interlocuteurs, Soté ne se prenait pas pour n'importe qui. Il rappelait qu'il était aussi incontournable que Benos alias Conforama, Boulou l'Agent immobilier ou Préfet, l'homme dont le nom était sur toutes les lèvres. Ceux-ci avaient besoin de ses services. C'était cette dépendance qui le rendait aussi vaniteux. (152)
\end{abstract}

Cette tranche narrative est substantiellement un autre portrait réalisé par le même et unique personnage-narrateur. Ce qu'elle a de plus que l'extrait précédent, c'est l'enchâssement inextricable des focalisations interne et externe. Et cette situation explique donc cette notion de "récit non-focalisé » qui voudrait simplement dire que tout y est en termes de vision du narrateur. Son regard comme sa voix, sa vision tout court, est omnisciente. Le personnage-narrateur sait tout, voit tout, sent tout et dit tout. Il plane sur l'existence du personnage de Soté dont il dresse un portrait miréaliste mi-subjectif. Ce personnage est sans doute ce que le narrateur dit de lui, seulement, l'amplification dans la vision des traits caractéristiques aussi bien physiques que moraux pousse le lecteur à soupçonner une certaine subjectivité du narrateur omnipotent, omniprésent et omniscient. Le mélange de visions narratives permet donc de créer certains bouts de récit où l'on recherche avec intérêt la position de celui qui voit, parle, entend et sait tout de l'univers à la fois purement fictif que réaliste dans sa tentative d'imiter le réel vécu. Dans les lignes qui suivent, nous entrons de plain-pied dans ce que la focalisation a d'intérêt littéraire, à savoir poétique et stylistique et qui est un atout considérable de ce roman avertisseur de la vraie face de l'immigration de citoyens du sud vers le nord, précisément des villes et villages africains vers la France et sa capitale, Paris.

\title{
2. Les paramètres de littérarisation de la vision narrative dans le roman
}

15 Cette étape se veut plus interprétative que descriptive afin de faire émerger du roman d'Alain Mabanckou la structure littéraire provoquée par le choix d'un personnagenarrateur enclin aux épanchements du cœur face au monde impitoyable qui s'offre à lui. La focalisation qui implique de grandes notions telles que l'énonciation, la narration ou la dichotomie datée du récit et du discours qui reste cependant opératoire pour des questions d'ordre méthodologique, est source de littérarité. Et selon que la vision du narrateur est interne comme c'est le cas tout au long de ce récit, les implications poétiques stylistiques sont importantes. Il en est de même pour les points de vue narratifs zéro et externe. À chaque acte précis d'énonciation, donc, produisant un énoncé type, correspondent des critères de poéticité ou de littérarité fournis par la langue d'écriture. 


\subsection{Du style réaliste du discours subjectif et du monologue introspectif}

16 Dans le roman Bleu-Blanc-Rouge d'Alain Mabanckou, la présence du sujet-narrateur désigné grammaticalement par le pronom de la première personne «je " est incompressible à tous les niveaux des différentes histoires ou descriptions qui s'enchâssent harmonieusement bien pour constituer ce récit dramatique et plaisant. Massala-Massala alias Marcel Bonaventure alias Éric Jocelyn-George est le personnage principal qui raconte l'histoire dont il est à la fois témoin et acteur clé. Il joue donc le rôle de narrateur principal de ce récit car tout ce que le lecteur apprendra sur l'univers romanesque, sur les pensées, comportements et paroles des personnages qui l'animent, est du ressort de ce personnage-témoin-acteur dans le déroulement de l'intrigue. Le narrateur est bien présent tout au long du récit et le passage suivant qui y est extrait n'en dit pas le contraire :

Moki était arrivé.

Ce que nous remarquions de prime abord, c'était la couleur de sa peau. Rien à voir avec la nôtre, mal entretenue, mangée par la canicule, huilée et noirâtre comme du manganèse. La sienne était blanchie à outrance. Il arguait que l'hiver y était pour quelque chose. Plus tard, en France, je sus qu'il s'appliquait sur tout le corps des produits à base d'hydroquinone. Les jeunes du pays qui s'ingéniaient dans leur cécité irréversible à singer les Parisiens, se rabattaient sur les produits bon marché fabriqués en Afrique comme les Ambi rouge ou Ambi vert. Le résultat n'était pas le même. Ils n'égalaient guère l'éclat de la peau d'un parisien. La chaleur suffocante du pays accélérait les effets secondaires. Les imitateurs écopaient d'allergies, de rougeurs et de caillots de sang sur le visage. (60-61)

Ce qui nous intéresse dans ce passage, ce sont les indices linguistiques qui marquent l'implication du narrateur dans son énoncé et qui font de lui le maitre de son énonciation. Il sait quand intervenir physiquement à travers l'usage du déictique « je » et tous les indices qui lui sont assujettis et où se placer de façon opportune dans l'énoncé. Ces indices de la subjectivité qui incarnent grammaticalement l'être du personnage-narrateur sont justement désignés par le concept de "subjectivèmes $»^{4}$. La première phrase de notre extrait "Moki était arrivé » peut donner lieu à toutes sortes de gloses pour appréhender la vision du narrateur. Cela se comprend aisément dès l'instant où cette phrase qui est tout un énoncé ne peut donner clairement, par rapport à elle-même, la position du narrateur. On sent bien qu'il est présent, qu'il s'est effacé mais sait tout et voit tout puisqu'il est la voix qui annonce l'arrivée de Moki au pays.

18 Ce petit énoncé sur lequel nous aurons à revenir plus en détail dans la dernière partie de notre réflexion est donc « vide » du point énonciatif, ce qui signifie, au contraire de ce que cette attribution peut laisser croire, qu'il se fonde sur l'enchevêtrement des trois visions narratives adoptées par un narrateur. L'emploi du pronom «nous ", la forme amplifiée du « je »+d'autres actants diffus, nous en donne une preuve. Et ce déictique à forme amplifiée est renforcée par le pronom possessif «la nôtre " qui désigne par humour noir du narrateur l'état de santé de la peau des hommes du pays qui, dit-il, était «mal entretenue, mangée par la canicule, huilée et noirâtre comme du manganèse ». L'exagération dépréciative qui sous-tend cet humour noir est en flagrante contradiction avec cette hyperbole que le même narrateur-témoin emploie pour décrire la peau du parisien : «La sienne était blanchie à outrance ». Deux mondes, deux couleurs différentes donc et pour le dire, un narrateur bien présent qui assume 
ses propos. La présence sans équivoque, cette fois-ci, de l'embrayeur « je », traduit bien cette expression de l'extérieur par les ressources de l'intérieur. Certains lexèmes comme les verbes de perception « remarquions ", « voir ", "sus » et ceux qui laissent entrevoir une opinion ou un jugement du narrateur comme "arguait", "s'ingéniaient", «singer", marquent bien une approche intime de la situation drolatique évoquée. Pour décrire cette situation avec le minimum de réalisme, le narrateur, comme dans la phrase à la vision "neutre ", à savoir "Moki était arrivé " située en tout début du passage, choisit une fois de plus le retrait de sa personne grammaticale de la syntaxe. Une posture qui n'est pas sans conséquence puisque nous avons annoncé le ton réaliste qui va envelopper la description de l'évènement burlesque qui est le suivant: «Les jeunes du pays qui s'ingéniaient dans leur cécité irréversible à singer les Parisiens, se rabattaient sur les produits bon marché fabriqués en Afrique comme les Ambi rouge ou Ambi vert. Le résultat n'était pas le même. Ils n'égalaient guère l'éclat de la peau d'un parisien. La chaleur suffocante du pays accélérait les effets secondaires. Les imitateurs écopaient d'allergies, de rougeurs et de caillots de sang sur le visage ». L'écriture est manifestement objectivée et le narrateur présente les faits tels quels, quitte au lecteur d'en tirer toutes les conséquences. Il ne s'agit plus seulement pour le personnage-narrateur Massala-Massala de rire simplement du contraste entre la condition des nationaux et celle de leurs frères expatriés, mais de traduire sans ambiguïté les effets pervers de l'illusion ou du rêve de l'espace « Paris » que vendent les seconds aux premiers. Un enchaînement de procédés stylistiques confirme ce ton à la fois réaliste et satirique. D'abord cette métaphore dépréciative "Les jeunes du pays qui s'ingéniaient dans leur cécité irréversible à singer les Parisiens, se rabattaient sur les produits bon marché fabriqués en Afrique comme les Ambi rouge ou Ambi vert ». Ensuite, ces phrases négatives qui donnent la nature du résultat dysphorique des singeries: «Le résultat n'était pas le même. Ils n'égalaient guère l'éclat de la peau d'un parisien ». Pour finir, et liée aux deux procédés précédents, en tant que chute du passage, cette hypotypose ${ }^{5}$ qui se fonde sur l'hyperbole et la gradation ascendante : «La chaleur suffocante du pays accélérait les effets secondaires. Les imitateurs écopaient d'allergies, de rougeurs et de caillots de sang sur le visage ».

19 À côté de ce type de récit qui mélange les visions narratives sous le couvert commode de la focalisation interne, il y a ces discours qui sont de véritables monologues dans lesquels la posture du sujet-narrateur est unique du début jusqu'à la fin du passage. Nous avons ci-dessous, une illustration de ces monologues introspectifs qui abondent dans le roman d'Alain Mabanckou :

Je suis Marcel Bonaventure, ça, je m'en souviendrai. Quoi qu'il m'advienne. Je ne peux plus le rayer de ma mémoire, ce nom. Je le porte comme je porte le nom de Massala-Massala. Je ne suis plus une seule personne. Je suis plusieurs à la fois. Quelqu'un prononcerait dans la rue le nom de Marcel Bonaventure? Je me retournerais. Comment gommer ce nom-là ? Il est en moi. C'est une question de dédoublement.

Je ne parle même pas de l'autre nom, Éric Jocelyn-George. Non, je ne voudrais pas brouiller l'intelligibilité de ma réminiscence. C'est assez confus comme ça sans parler de ce troisième nom : Éric Jocelyn-George. C'est encore moi. Moi, MassalaMassala. Chaque nom a son histoire. Chaque nom est une période, un fait de mon existence.

Où sont-ils, ceux de notre milieu?

Où sont-ils donc?

Je tâte les murs du silence. Pourquoi n'entends-je que l'écho de leur voix? Ils avaient tous des ailes pour s'envoler lorsque la pierre, jetée par un enfant 
turbulent, était tombée en plein milieu de la cour où nous nous disputions un morceau de pain comme des oiseaux. J'ai voulu m'envoler, moi aussi. Je n'étais qu'un oisillon. On ne vole pas d'un seul coup sans appréhender le vide, la force de la pesanteur. Il faut procéder par étapes. Gravir les marches de la patience. Étirer d'abord les ailes, les battre ensuite dans le sens du vent. Recroqueviller les pattes et quitter le nid pour un premier envol. Je n'étais qu'un oisillon. J'ai volé par mimétisme.

Voilà pourquoi je me suis retrouvé ici... (127-128) existence. Ce passage est important parce qu'il présente le retour psychologique du personnage sur la question de son identité véritable et sur sa vie civile d'avant la situation dans laquelle il se trouve au moment où il énonce cette tranche de discours. Cette situation est celle du détenu aux trois identités, en attente de rapatriement au pays natal après l'amère expérience de l'espace «Paris». Ces trois identités sont: « Marcel Bonaventure », « Éric Jocelyn-George » et « Massala-Massala ». Le dernier nom du narrateur est le vrai, l'original et les deux autres, des faux qui induisent clairement la vie illusoire qu'il a dû mener à un moment de son itinéraire existentiel. On le note bien, l'introspection du narrateur à la triple identité n'est pas une méditation métaphysique. Au contraire, elle est empreinte de réalisme parce que la vision du narrateur, en dépit de son caractère interne ou subjectif, se fonde sur des faits qui reflètent bien la désillusion des immigrés noirs dans l'univers étranger et hostile de l'Occident. Un ensemble de lexèmes peuvent servir à former le champ lexical du souvenir de cet espace. Ce sont: d'une part le verbe de perception conjugué à un futur de type atemporel "souviendrai", une preuve de ce que le narrateur médite sur des faits passés qu'il n'oubliera jamais, et d'autre part, le lexique du souvenir constitué par les lexèmes suivants : « mémoire », « réminiscence ", « histoire », " période », « fait de mon existence ». Et le retour du personnage sur les évènements douloureux qui ont émaillé son bref séjour à Paris, capitale de la métropole française et qui lui ont valu de brader son identité réelle pour survivre, ce retour de la conscience sur elle-même, disons-nous, s'exprime aussi à travers des interrogations oratoires du type "Comment gommer ce nom-là ?». Cette même question oratoire reste valable pour la deuxième fausse identité, celle d'Éric Jocelyn-George. D'autres indices de la langue permettent de construire le cadre de l'introspection autour $\mathrm{du}$ « je » narrateur. Ce sont entre autres l'emphase et les phrases à verbe de sémantisme vide ${ }^{6}$ telles que "Je suis Marcel Bonaventure, ça, je m'en souviendrai », «Je ne peux plus le rayer de ma mémoire, ce nom » et « Éric Jocelyn-George. C'est encore moi. Moi, Massala-Massala ». Le deuxième niveau de l'introspection du héros malheureux est centré sur le sentiment de solitude qui le ronge et qui est traduit prioritairement par deux procédés de style. D'abord, les interrogations rhétoriques à travers lesquelles le sujet-narrateur prend conscience de sa solitude, abandonné par tous : « Où sont-ils, ceux de notre milieu ? Où sont-ils donc? Je tâte les murs du silence. Pourquoi n'entends-je que l'écho de leur voix? ». Ensuite, il y a surtout cette vaste allégorie de l'oisillon abandonné de tous les autres oiseaux face au danger, qui peut être interprétée comme la lâcheté des complices, professionnels de la magouille et des arnaques, qui se sont tous évanouis dans la nature pour laisser le débutant à son propre sort. Cette allégorie construite autour des métaphores, des aphorismes et gradations ascendantes est celle-ci :

Ils avaient tous des ailes pour s'envoler lorsque la pierre, jetée par un enfant turbulent, était tombée en plein milieu de la cour où nous nous disputions un morceau de pain comme des oiseaux. J'ai voulu m'envoler, moi aussi. Je n'étais 
qu'un oisillon. On ne vole pas d'un seul coup sans appréhender le vide, la force de la pesanteur. Il faut procéder par étapes. Gravir les marches de la patience. Étirer d'abord les ailes, les battre ensuite dans le sens du vent. Recroqueviller les pattes et quitter le nid pour un premier envol. Je n'étais qu'un oisillon. J'ai volé par mimétisme (128).

21 La chute de ce tableau à l'allure d'une fable est juste dans cette petite phrase emphatique qui met en relief le sujet en proie à un espace carcéral que le lecteur n'a pas du mal à décoder: "Voilà pourquoi je me suis retrouvé ici... ». Une analyse sommaire de cette chute donne ceci : «pourquoi»= la causalité ou la justification; « suis retrouvé » = condition, situation; « je / me » = sujet-narrateur ; «Voilà / ici »= espace carcéral (la prison). En résumé, le « je » narrateur plonge dans une introspection parce qu'il se sait coupable d'usurpation d'identité et paie de sa détention pour tous ses complices qui se sont volatilisés dans la nature. Le style de cette introspection est tout simplement réaliste parce que bâti sur la vraisemblance.

\subsection{La fonction rythmique de la focalisation dans Bleu-Blanc-Rouge}

22 Cette dernière étape de notre réflexion va nous permettre d'aborder un aspect important de la vision narrative. Le romancier a laissé à son personnage-narrateur le soin de construire son récit, ce que ce dernier a bien réussi autour de sa personne par le choix de certaines stratégies narratives selon un ordre rythmique et qui, de ce fait, rendent le récit très vivant. La première facette de cette fonction rythmique est saisissable dans la construction cinématographique de ce roman qui fonctionne comme un film à deux grands épisodes contenant chacun des intrigues bien enchâssées, les faits des unes justifiant bien ceux des autres et vice-versa.

23 Ainsi, pareil au roman ou un film d'enquêtes policières, le roman d'Alain Mabanckou débute par une «Ouverture » qui est en réalité son épilogue (ou la fin du roman ou du film) qui précède l'intrigue à proprement dite et dont la chute correspond à la «Fermeture » qui vient confirmer les faits de la fin évoqués par anticipation dans l' « Ouverture » en question. Le récit du sujet-narrateur s'ouvre donc par un flashback, c'est-à-dire un retour en arrière qui, en littérature, porte le nom d'analepse. Ce procédé rhétorique est lui-même, et de toute évidence, subjectif parce que s'inscrivant dans le cadre d'un monologue intérieur dans lequel le locuteur exprime à la fois ses regrets, ses angoisses et son désir ardent de s'en sortir. Se sortir de quoi ? Le lecteur, à ce stade, comme dans le roman policier ou le film du même type qui débute par un flashback, ignore tout de l'intrigue qui pousse le personnage-narrateur dans cette situation d'examen personnel de conscience. La réponse à la question se trouve dans les deux grandes séquences (ou épisodes) intitulées «Le pays » (29) et "Paris » (119) et dont les péripéties tissées harmonieusement trouvent leur clôture dans la "Fermeture » de l'œuvre dont la fin de cet épilogue - et c'est là l'un des intérêts du rythme de la vision narrative du roman - se trouve dans l' "Ouverture» ou prologue par analepse. Le rythme de la narration fonctionne, sans conteste, en boucle. Le lecteur part de la fin du récit pour comprendre les raisons du sujet-narrateur interrogeant sa condition d'existence pour finir par un épilogue dont la suite se trouve, contre toute attente, dans le prologue ou « Ouverture ». La vision narrative dans le roman d'Alain Mabanckou est originale. Le schéma suivant permet de rendre compte de ce rythme de premier niveau, externe à l'énoncé mais, qui assure sa matérialité et sa fonctionnalité. 
Schéma 1 : le rythme externe de la vision narrative

Alain Mabanckou

Roman (Première partie) (Deuxième partie)

Bleu-Blanc-Rouge Ouverture « Le Pays » « Paris » « Fermeture »

(« Je »-sujet-Narrateur)

Massala-Massala alias (Moki et son retour (Rue du Moulin-vert (Paris 14 ${ }^{\mathrm{e}}$ )

Marcel Bonaventure L'ombre de Moki Marcel Bonaventure

alias Éric Jocelyn-George Le père de Moki Éric Jocelyn-George

Le Général de Gaulle Château-Rouge (Paris $18^{\mathrm{e}}$ )

La villa blanche L'agent immobilier

Les taxis L'italien

La légende des aristocrates Conforama

Le nouveau-né Le piocheur

Paris est un grand garçon) Préfet

La Seine-Saint-Denis

Juste à côté de ce rythme externe dont le mouvement se développe en boucle, nous avons de nombreux indices qui participent de cette même vitalité du récit dans le roman d'Alain Mabanckou. Seulement, ces indices ne sont pas donnés systématiquement comme dans la structure externe du rythme. Il faut les interroger dans l'énoncé pour les faire apparaitre en surface. Nous avons, entre autres, ce type de syntagmes qui servent tantôt d'amorce tantôt de synthèse aux différentes portions narratives, et cela à travers tout le roman, le dédoublement de la voix narrative et le rythme de la phrase.

Dans le récit du sujet-narrateur, il existe ce type de syntagmes qui accomplissent une fonction d'amorce ou de clôture des différentes séquences narratives. Il s'agit la plupart du temps de syntagmes dont le rythme est aussi bien unitaire, binaire que ternaire. Chacun de ces syntagmes repose donc sur un, deux ou trois unités de sens. Bien mieux, leur type et leur forme grammaticale sont variables d'une phrase à une autre. Nous allons nous intéresser à ces syntagmes dont les formes sont récurrentes dans l'énoncé. Nous allons nous saisir, pour cela, de l' "Ouverture » comme support pour y extraire les illustrations nécessaires à l'analyse de ces syntagmes contribuant à la structure rythmique du récit. Cette analyse que nous ferons vaut pour tout le reste du récit et de sa "Fermeture » qui est d'ailleurs une réplique de cette "Ouverture ». Ainsi, comme incipit de la toute première étape de l'énoncé, ce syntagme au rythme unitaire "J'arriverai à m'en sortir » (11) qui annonce des faits dont le cours de la narration va varier avec cette autre expression du même type que la précédente « Les choses vont se précipiter » (12). De ce syntagme à valeur d'enchaînement à celui-ci : «Oui, la porte qui se referme » (13), il court un temps du récit qui s'efface pour faire place à un autre au moment où le personnage-narrateur confirme son arrestation pour faux et usage de faux à travers toujours un syntagme au rythme unitaire "Ils ont vaincu " (13). Toute une série de faits que le sujet-narrateur explique de l'intérieur, qui le culpabilisent, et la brève conclusion de ces faits qui fait écho aux syntagmes introductif est rythmiquement binaire : "je suis resté // là » (18), l'essentiel de l'information étant porté par le déictique «là » désignant la prison. Ces informations disent simplement que le malfrat (le personnage-narrateur lui-même) a bien été arrêté par la police et qu'il sera détenu pendant un certain temps avant son rapatriement au pays natal. Ces différentes portions narratives constituent donc la substance de ce long monologue qui 
sert d'« Ouverture " atteignant à certains endroits, à une introspection profonde : "Une question me traverse encore l'esprit » (13). Tout en s'appropriant cette question qui lui permet de faire un retour introspectif sur les conditions de son arrestation, le sujet-narrateur n'est pas pour pourtant coupé de la réalité ambiante du moment, celle de son transfèrement à la prison dont le lieu lui est totalement inconnu : «Mais nous roulions encore » (19). Et durant tout le trajet de non-retour à la maison, le sujetnarrateur reste plongé dans le questionnement interne de sa conscience. Il finit par se convaincre de ce que des aspects de sa mésaventure lui échappent : " Plusieurs choses » (21). S'il en est ainsi, c'est parce que tout lui semble confus et sa condition de prisonnier en plein transfèrement le prive de tous ses droits les plus élémentaires même celui de la parole : «Silence» (21). Cette situation est un signe d'impuissance et de résignation qui s'exprime à travers ce syntagme performatif «Et attendre» (22). Finalement, l'attente ne sera point déçue puisqu'il sera jeté dans une cellule dans laquelle il va cependant faire un examen intuitif des lieux « il y avait donc une vie dans la bâtisse » (23). Il y a ici une antithèse blottie dans le rapport entre les termes " vie » et «bâtisse ». Cette figure, d'un point de vue sémantique, et en termes de conversion des contraires qui s'opère en toute instance dialectique, est le lieu de neutralisation ou d'euphémisation du caractère impitoyable de la détention dont la «bâtisse » est le symbole. Il existe un petit espoir d'appartenance au monde malgré tout qui demeure chez le prisonnier. C'est donc le temps de la détention qui renforce son introspection dans une geôle qu'il décrit à travers la figure de l'aposiopèse comme un enfer: «L'odeur du moisi... » (24). Aussi, en dépit de son esprit tourmenté, le narrateur s'est-il souvenu du vrai responsable de ses frasques et peut donc s'écrier, à travers ce court syntagme à verbe de sémantisme vide pour faire surgir l'identité du vrai coupable : «C'est Moki » (25). Le sujet-narrateur, à juste titre, en veut terriblement à ce dernier et son introspection va être interrompue par cette question nécessairement rhétorique renforcée par la figure de l'aposiopèse: «Où es-tu à présent ?...» (26). De toute évidence, le personnage-narrateur n'aura aucune réponse à cette question relevant de sa réminiscence ou de son examen de conscience dont les derniers faits se trouvent suggérés à travers une autre phrase à verbe de sémantisme vide qui projette la condition du personnage narrateur dans l'imagination du lecteur : «Ce qui importe à ce stade, c'est de comprendre » (27).

26 Comme on peut le constater, cette "Ouverture", à l'instar de tout le récit, est structurée par des syntagmes marqués qui assurent la vitalité ou le dynamisme du rythme sous-tendant la narration. Toutes ces expressions ont pour point commun d'être très concises, de se positionner comme éléments d'amorce ou de synthèse et de participer à la structuration de l'examen de conscience de Massala-Massala alias Marcel Bonaventure alias Éric Jocelyn-George, le sujet-narrateur. Quant aux différences, elles se situent du point de vue grammatical : les syntagmes s'alignent chacun sur l'une de ces structures profondes suivantes: Sujet+Verbe+ Complément ("J'arriverai à m'en sortir» (11); Sujet+ Verbe («Les choses vont se précipiter» (12); Groupe Nominal («Plusieurs choses» (21); Nom (« Silence » (21); Verbe (« Et attendre» (22). Le rythme varié produit par ces phrases dont le sémantisme révèle l'introspection du sujetnarrateur est expressif de son état d'angoisse.

27 Quant aux types de phrases et à leurs formes grammaticales, nous avons le type déclaratif de forme affirmative («je suis resté là » (18), de forme emphatique («Ce qui importe à ce stade, c'est de comprendre » (27); le type interrogatif renforcé par la suspension «Où es-tu à présent ?...». Tous ces indices syntagmatiques associés aux 
paradigmes nominaux répertoriés supra donnent un rythme varié au récit et la vision du sujet-narrateur y est à la fois interne qu'externe ou omnisciente. La voix qui raconte donc les faits est tantôt identifiable et localisable dans certains énoncés, tantôt absente dans d'autres comme si ceux-ci ne résultaient pas de sa narration. Si le premier cas de la narration interne prend en compte les phrases dont les prédicats on pour sujet le «je» énonciatif ou les phrases interrogatives ou ponctuées par la suspension, la narration non-focalisée, elle, prend en compte tous les syntagmes à rythme unitaire, c'est-à-dire à une seule unité de sens. Cette posture narrative qui fluctue d'une tranche narrative à une autre confère à ce grand monologue qu'est l' "Ouverture " le caractère d'un véritable moment de prise de conscience du sujet-narrateur de sa condition difficile de malfrat mis aux arrêts pour n'avoir été, au cours de son bref séjour parisien, qu'un faussaire parmi tant d'autres, plus chanceux et plus expérimentés.

Quant au dédoublement de la voix narrative dans le récit, elle est avec le rythme de la phrase deux éléments intéressants de la fonction rythmique qu'il convient d'interroger. Dans Bleu-Blanc-Rouge de Mabanckou, le dédoublement de la voix narrative est un moyen sûr de rendre dynamique le rythme du récit. Celui-ci découle, d'une part, de la prise de parole des autres personnages qui, comme le personnage-narrateur principal, assument leur présence dans les énoncés qu'ils produisent et d'autre part, d'un cas très intéressant d'intertextualité. D'abord, le premier cas de dédoublement où un personnage autre que le sujet-narrateur attitré, en la personne de Massala-Massala, raconte des faits mais dans la narration englobante de la voix principale. Il y a, pour ainsi dire, des voix narratives secondaires intriquées dans une voix narrative de premier niveau. Une illustration intéressante de ce procédé narratif nous est donnée dans les intervalles des pages suivantes : 73-87 (Moki raconte son épopée glorieuse de sapeur ${ }^{7}$.) ; 102-104 (Le père du sujet-narrateur Massala-Massala s'en prend vertement à son fils qui nie être l'auteur de la grossesse d'Adeline.); 135-136 (Moki explique au nouveau parisien désabusé les réalités impitoyables des expatriés.) ; 162-164 ( Préfet se présente en mécène au sujet-narrateur.) ; 166-167 (rappel à l'ordre du « bleu » par Moki pour prévenir toute renonciation.) ; 170-174, 183-184 et 187-188 (Initié au faux et à l'usage du faux par Préfet, le personnage-narrateur agit sous les ordres de son employeur.)

\section{Schéma 2 : la structure enchâssée de la narration}

Énoncé de la voix narrative

principale

Si l'on applique la lecture sémiostylistique, précisément la lecture actantielle à ces différentes tranches narratives qui sont autant de prises de paroles de certains personnages, il ressort qu'on peut leur appliquer une représentation schématique unique. Les informations à utiliser pour renseigner le schéma seront: au niveau $\alpha$, l'Émetteur $\alpha$ (le scripteur Alain Mabanckou), le Récepteur $\alpha$ (nous les lecteurs en chair et en os); au niveau actantiel I, le Narrateur I (le sujet-narrateur Massala-Massala), le récepteur I (le lecteur); au niveau II, l'Émetteur II (Moki, le père du sujet-narrateur, Préfet), le Récepteur II ( en ordre: l'assistance avec pour répondant immédiat à l'émetteur, tous ceux qui dans l'assistance posent des questions; le sujetnarrateur pour tous les émetteurs signalés ci-dessus. Tentons une schématisation du système actantiel valable pour toutes ces prises de paroles intégrées au récit du sujetnarrateur principal telle qu'on peut trouver des modèles similaires dans le livre de 
Molinié et Viala: Approches de la réception, sémiostylistique et sociopoétique de Le Clézio8. L'énoncé 1 de Moki est choisi à titre illustratif dans l'ensemble des énoncés :

Schéma 3 : le système actantiel de l' énoncé de Moki

E 2 Moki

répondants

II E $1 \mathrm{R}$

Moki assistance (répondants)

I E ( ) R

narrateur lecteur

$\alpha \mathrm{ER}$

scripteur

L'enchevêtrement réussi des tranches narratives produites par des personnages autres que le sujet-narrateur contribue bien au dynamisme du rythme externe. Cette facette du rythme généré par la focalisation est aussi intéressante dans les contextes du style de discours et d'intertextualité, comme dans la réponse de l'oncle de Massala-Massala quant à la contribution de ce dernier pour le faire partir en France, précisément à Paris :

Je calmai mon père qui s'imaginait que Moki s'était opposé à mon départ pour la France. Je n'avais de toutes les façons pas mon billet, même si mon oncle avait laissé entendre, dans cette langue entortillée qui était la sienne, qu'il avait «un préjugé favorable de contribution à cette initiative fort courageuse mais qui restait à discuter, tout au moins dans son fondement, sans pour autant remettre en cause l'ensemble de l'échafaudage ». Mon père n'avait rien saisi et était revenu sans être renseigné sur la position de mon oncle, tant cette prose égarait sa lucidité. (96)

Il est évident qu'il y a ici une intrication de deux discours de style différent, le premier en rapport d'hyperonymie avec le second est le style indirect libre employé par le sujetnarrateur principal. À l'inverse, et en rapport d'hyponymie avec ce premier type de discours, le discours indirect qui permet au personnage-narrateur de rapporter les propos de son oncle quant à son aide financière. Du point de vue typographique, le discours indirect libre, discours intégrant, est en caractère d'imprimerie ordinaire et le discours indirect intégré, rapportant la réponse ambiguë de l'oncle, lui, est écrit en italique. Cette technique d'imbrication des styles de discours les uns dans les autres permet de rythmer le cours de la narration et de casser la monotonie du récit procédant du seul rapport du « je » tout puissant à son énoncé afin de rendre celui-ci plus vivant. Il en est de même de cette lettre type destinée à tous les Parisiens qui voulaient créer l'illusion de la réussite et faire rêver les filles restées au pays. Cette lettre au ton comique est située à la page 133 du roman et selon les dires du personnage-sujet, son auteur est anonyme : "Qui l'avait rédigée pour le plaisir et le bonheur de la communauté? Je ne le savais pas. Ceux qui la recopiaient ne remplaçaient que le prénom de la destinataire » (132), à savoir « Marie-Josée » (133).

Cette lettre en effet, crée un effet de dédoublement de la voix narrative dans l'énoncé. Il y a une subordination d'un « je » sujet-narrateur à un autre, situé au cœur de la lettre et qui s'adresse à une dulcinée restée au pays et que l'auteur toujours anonyme veut impressionner ou séduire à distance. Le caractère inépuisable de l'autorité de la lettre, $\mathrm{du}$ « je » narrateur toujours ressuscité, du temps de l'énonciation et de l'énonciateur aussi éternel que le temps de l'utilisation anonyme de la missive, c'est-à-dire de l'énoncé reste invariable. L'intrusion de cette lettre à usage collectif dans le récit du 
personnage-narrateur lui permet de varier quelque peu le rythme de la narration qui, ainsi, passe d'un ton dramatique à un autre, comique voire burlesque. Nous retranscrivons cette lettre type qui, au-delà du personnage-narrateur omniscient, met en relief le génie de l'auteur, en chair et os, de Bleu-Blanc-Rouge, à savoir Alain Mabanckou :

Ma chère Marie-Josée

Je t'écris en face de la tour Montparnasse que je contemple chaque matin depuis la salle de bains de notre magnifique appartement du quatorzième arrondissement. L'été vient de s'achever sur la plus belle ville du monde. Nous allons vers l'automne, pour ensuite admirer la splendeur blanche de la neige en hiver.

Je t'ai acheté beaucoup de cadeaux, des vêtements de grands couturiers du faubourg Saint-Honoré. Je t'ai aussi acheté une paire de mocassins Weston. Je veux bien te les envoyer mais je crains que tu ne fasses la java avec mes adversaires locaux, des gens qui ne savent même pas combien coûte un pantalon Yoshi Yamamoto. Moi je n'ai plus rien à prouver. Je suis un Parisien avec un grand $\mathrm{P}$.

J'ai survolé le ciel en avion pendant une nuit entière et j'en ai même profité pour faire mes besoins alors que nous planions sur le pays, chose qui n'est pas donnée à n'importe qui, surtout pas aux paysans. C'est dire que j'ai nourri les poissons de notre océan Atlantique. Je compte t'épouser pour le meilleur seulement, il n'y aura pas de pire avec moi. Je te donne ma parole de Parisien. Compte sur moi, je prépare notre avenir. Je t'embrasse tendrement. Je t'aime, ma petite Golden (c'est comme ça qu'ils appellent le genre de pommes que j'aime ici)...

Ton fiancé parisien.

Ici aussi, la typographie est un repère qui aide à identifier l'hypertexte de l'hypotexte dans le contexte de l'intertextualité qui opère ici. Cette lettre est écrite en italique et se distingue nettement de l'ensemble narratif dans lequel elle a été incorporée et qu'elle rythme ainsi de par son surgissement dans l'ensemble en question. Ainsi, en analysant ce rythme produit par l'intertextualité, on voit comment le narrateur opère une dédramatisation de sa narration construite autour de la thématique de la condition d'existence médiocre des Africains immigrés en Europe

Au terme de nos propos, il ressort que la focalisation, pour être appréhendée nécessite l'analyse de certaines composantes du texte littéraire telles que la narration, l'énonciation, la tonalité réaliste, le rythme, cette catégorie du texte narratif est génératrice de littérarité ou de poéticité. Selon le point de vue adopté par le narrateur dont le statut par rapport aux personnages, sujets ou actants compte dans l'analyse, le discours développe des stratégies à la fois langagières, formelles et thématiques qui atteignent à sa littérarisation. Le roman Bleu-Blanc-Rouge d'Alain Mabanckou qui a privilégié le statut de sujet-narrateur en tant que celui-ci est l'instance énonciatrice porteuse du projet discursif du roman constitue un vaste discours introspectif dans lequel les enjeux d'analyse théorique et méthodologique sont importants. Il soulève la réflexion à la fois sur les questions de l'énonciation mixte, du mélange des genres et des formes littéraires, du réalisme dans le discours, des formes-sens, du rythme externe, etc. Pour avoir confié la narration à un regard ou une voix de narrateur qui, tantôt intègre son histoire en tant que personnage principal, tantôt s'éclipse pour libérer son récit de la subjectivité et des commentaires personnels, tantôt se donne tous les droits de narrateur pour prendre contrôle de tous les personnages, y compris lui-même, Mabanckou nous gratifie d'un récit vivant dans lequel s'épuisent tous les points de vue narratifs et donc plusieurs histoires. 


\section{BIBLIOGRAPHIE}

Barthes, Roland, Le degré zéro de l'écriture, Paris, Seuil, 1972.

Borgomano, Madeleine, « le personnage-narrateur ... », Variations sur le personnage, Abidjan, CEDA, 1985, p. 21-36.

Fromilhague, Catherine et Sancier-Château, Anne, Introduction à l'analyse stylistique, Armand Colin, 2016.

Genette Gérard, Figures III, Paris, Seuil, 1972.

Jouve, Vincent, La poétique du roman, Paris, Armand Colin, 2001.

Kerbrat-Orecchioni, Catherine, L'énonciation, de la subjectivité dans le langage, Paris, Armand Colin, 1980.

Kundera, Milan, L'art du roman, Gallimard, 1986.

Molinié, Georges et VIALA Alain, Approches de la réception..., Paris, PUF. 1993.

Molinie, Georges, La stylistique, Paris, PUF, 1993.

Reboul, Olivier, Introduction à la rhétorique, Paris, PUF, 1991.

Todorov, Tzvetan et Ducrot, Oswald, Dictionnaire encyclopédique des sciences du Langage, Paris, Seuil, 1972.

\section{NOTES}

1. Dans son livre Figures III, Seuil, 1972, p. 239.

2. Cette expression est empruntée à Gérard Genette, dans son ouvrage Figures III, Seuil, 1972, p. 206, pour traduire le phénomène énonciatif de la vision panoramique ou surplombante du narrateur omniscient et omnipotent qui, en véritable créateur, ne perd jamais le contrôle de l'univers et de ses êtres dont il relate, les réalités par le menu.

3. Pour Gérard Genette, le "récit non-focalisé » désigne le récit classique caractérisé par une absence de focalisation correspondant bien à la focalisation dite zéro.

4. Par ce concept, Kerbrat-Orecchioni désigne les unités de la subjectivité : «il va de soi que toute unité lexicale est [...] subjective, puisque les "mots" de la langue ne sont jamais que des symboles substitutifs et interprétatifs des "choses" » (L'énonciation, de la subjectivité dans le langage, 1980 :70). 5. Olivier Reboul définit l'hypotypose comme étant la figure qui consiste à décrire un spectacle ou un évènement de façon si vivante que l'auditoire (ici le lecteur) croit l'avoir sous les yeux ". Consulter pour cela «L'index et glossaire des termes techniques» de l'ouvrage Introduction à la rhétorique, PUF, 1991, p. 239.

6. Fromilhague et Sancier-Château définissent ce type de phrase à «valeur picturale » comme étant celui où « le noyau verbal pose seulement l'existence de la réalité évoquée, [où] la phrase s'engage à partir de c'est, il y a ou voici ». Aussi, dans la phrase à verbe de sémantisme vide, « le procès ou l'état dépeint se fixe dans l'imagination du lecteur » (Introduction à l'analyse stylistique, $2016: 170)$.

7. Une note infra-paginale de l'auteur définit le Sapeur comme étant une "personne se reconnaissant et reconnue par ses pairs comme appartenant à la SAPE (Société des ambianceurs et des personnes élégantes » (74). 
8. Publié aux Éditions PUF, en 1993. Consulter ce livre, de la page 47 à la page 136 pour retrouver de nombreux schémas actantiels illustratifs.

\section{RÉSUMÉS}

Dans le roman Bleu-Blanc-Rouge d'Alain Mabanckou, la focalisation est une sémiose appréhendable à travers la pragmatique d'un «je-moi-narrateur» qui raconte sa présence au monde. La narration acquiert de fait sa littérarité par le croisement de faits discursifs d'extra et d'intra diégétique d'avec ceux du diégétique pour construire le récit rythmé d'un mondain offert à la méditation.

In Alain Mabanckou's novel Bleu-Blanc-Rouge, focusing is a semiotic which can be understood through the pragmatics of an "I-me-narrator" who tells of his presence in the world. Narration in fact acquires its literarity by the intersection of discursive facts of extra and intra diegetic with those of the diegetic to construct the rhythmic narrative of a mundane offered for meditation.

\section{INDEX}

Mots-clés : roman, focalisation, sujet-narrateur, littérarité, rythme

Keywords : novel, focalisation, subject-narrator, literarity, rhythm

\section{AUTEUR}

\section{ABOUBAKAR GOUNOUGO}

Université Félix Houphouët-Boigny, Côte d'Ivoire 